\title{
Approximate Engineering Solution for Predicting Groundwater Table Variation During Reservoir Drawdown on the Basis of the Boussinesq Equation
}

\author{
Jianping Sun, Ph.D. ${ }^{1}$; Jiachun Liं ${ }^{2}$ Q Qingquan $\mathrm{Liu}^{3}$; and Huiqin Zhang, Ph.D. ${ }^{4}$
}

\begin{abstract}
With reservoir drawdown, the groundwater table in the adjacent aquifer falls down correspondingly. It is useful to calculate the groundwater table variation as a function of time during reservoir drawdown for hydraulic and hydrological purposes. The Boussinesq equation with a moving boundary is applied to analyze the groundwater table variation in the unconfined aquifer during reservoir drawdown. This approach assumes a negligible seepage face. Because the moving boundary condition in the mathematical formulation precludes analytical solutions even for the linearized Boussinesq equation, we have transformed the Boussinesq equation into an advection-diffusion equation to address the negligible seepage face and the moving boundary condition. On the basis of the Laplace transformation, we yield an analytical solution of a fixed boundary problem, which is further simplified to upper and lower polynomial solutions for convenient practical use. The polynomial approximate solutions are satisfactorily compared with a number of numerical simulations of the nonlinear Boussinesq equation. The results indicate that the polynomial solutions match well with the numerical solution, but demonstrate that the replacement of the sloped reservoir-aquifer interface by a vertical interface may cause errors of up to $10 \%$ of the height of the reservoir drawdown in the prediction of the groundwater table location. On the basis of the polynomial solutions, a methodology is provided to determine the ratio of hydraulic conductivity to specific yield along with a chart for convenient practical use. The limitation of the present study is that the presented solution tends to underestimate the groundwater table with seepage face neglected for rapid drawdown, high specific yield, low hydraulic conductivity, or mildly sloped interface cases. DOI: 10.1061/(ASCE)HE.1943-5584.0000372. (C) 2011 American Society of Civil Engineers.
\end{abstract}

CE Database subject headings: Hydrologic models; Groundwater management; Water table; Boussinesq equations; Aquifers; Verification; Reservoirs.

Author keywords: Hydrologic models; Water table; Boussinesq equations; Aquifers; Verification.

\section{Introduction}

During reservoir drawdown, the groundwater table in the adjacent aquifer falls. The prediction of groundwater table variations in the aquifer during reservoir drawdown is useful for hydraulics and hydrological analysis. The Boussinesq equation is most often used to analyze groundwater flows in an unconfined aquifer (Mizumura 2009; Bansal and Das 2010). Analytical solutions for this equation can provide insight into the physics of groundwater flows. In the meantime, they can also be used to verify numerical solutions. Therefore, it is still meaningful for us to explore a new approximate analytical solution for extending the existing solutions and providing additional applications.

\footnotetext{
${ }^{1}$ Institute of Mechanics, Chinese Academy of Sciences, Beijing 100190, China. E-mail: sunjp@ntu.edu.sg

${ }^{2}$ Professor, Institute of Mechanics, Chinese Academy of Sciences, Beijing 100190, China (corresponding author). E-mail: jcli05@imech .ac.cn

${ }^{3}$ Professor, Institute of Mechanics, Chinese Academy of Sciences, Beijing 100190, China. E-mail: qqliu@imech.ac.cn

${ }^{4}$ Institute of Mechanics, Chinese Academy of Sciences, Beijing 100190, China. E-mail: zhanghuiqin@imech.ac.cn

Note. This manuscript was submitted on May 18, 2010; approved on January 10, 2011; published online on January 12, 2011. Discussion period open until March 1, 2012; separate discussions must be submitted for individual papers. This paper is part of the Journal of Hydrologic Engineering, Vol. 16, No. 10, October 1, 2011. CCASCE, ISSN 1084-0699/ 2011/10-791-797/\$25.00.
}

The Boussinesq equation is nonlinear and hardly has theoretical solutions in general. In previous works, the equation was analytically solved for specific initial and boundary conditions (Telyakovskiy and Allen 2006; Telyakovskiy et al. 2010). Hogarth et al. (1999) used the Boussinesq equation to describe groundwater flows in an initially dry aquifer when the level of a stream experiences temporal variation. Analytical solutions on the basis of the Heaslet-Alksne technique (Heaslet and Alksne 1961) were given to estimate the groundwater table height and flux between a stream and the adjacent aquifer. Parlange et al. (2000) reported a useful analytical solution of the Boussinesq equation that provides an insight into the physical processes of water exchange between an aquifer and a free water body of varying elevation. The solution is able to describe water movement both in and out of the aquifer, including the case of a finite aquifer. Lockington et al. (2000) presented a simple analytical solution of the Boussinesq equation for water motion from a stream into an initially dry, unconfined, horizontal, semi-infinite aquifer on the basis of Shampine's transformation when the boundary condition obeys a power law of time. Song et al. (2007) proposed a perturbation solution of the Boussinesq equation for one-dimensional tidal groundwater flows in a coastal unconfined aquifer. This solution exhibits various propagation characteristics of tidal fluctuating signals in the aquifer. Serrano et al. (2007) solved the Boussinesq equation by using the decomposition method to model transient stream-aquifer interaction, and gave some analytical solutions subjected to transient, largeamplitude, periodic boundary conditions. However, all of the previously mentioned researchers assumed that the interface between 


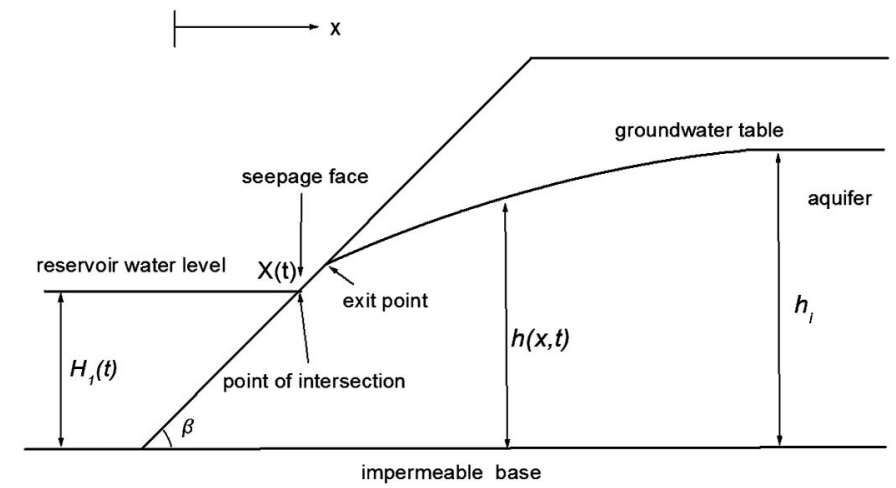

Fig. 1. Sketch of idealized cross section for the mathematical model (seepage face is neglected if the exit point coincides with the intersection as in this study)

the free water body and the adjacent unconfined aquifer was vertical.

In a natural reservoir-aquifer system, the interface between the reservoir and the adjacent aquifer is generally nonvertical, which results in a moving boundary condition in the mathematical model for groundwater table prediction during reservoir drawdown (see Fig. 1). This moving boundary precludes analytical solutions even for the linearized Boussinesq equation. Only a few studies have addressed the issue of the moving boundary condition. Nielsen (1990) presented an analytical investigation in which the assumption of a fixed boundary condition was relaxed. He derived a perturbation solution for small-amplitude fluctuations in the water table on the basis of the linearized Boussinesq equation by matching a prescribed series solution with the moving boundary condition. Li et al. (2000) transformed the Boussinesq equation into an advection-diffusion equation with an oscillating water table to account for the moving boundary effect. Maintaining the simplicity of the linearized Boussinesq equation, they presented a new perturbation approach to deal with the propagation of spring neap tides. However, all of the foregoing solutions focus on the sinusoidal oscillating moving boundary condition and cannot be applied directly to study the effects of reservoir drawdown with constant speed on groundwater table variation in the adjacent aquifer.

The purpose of the present paper is to extend the work of Li et al. (2000) to determine the groundwater table location during reservoir drawdown. A new analytical solution of the linearized Boussinesq equation is presented to describe groundwater table variation in a semi-infinite unconfined aquifer when reservoir water drops at a constant speed. On the basis of the analytical solution by virtue of the Laplace transformation, we yield the upper and lower bound solutions, which are further simplified into an approximate polynomial formula. They are well verified by comparison with a number of numerical simulations of the nonlinear Boussinesq equation for different physical parameters. Finally, a methodology along with a chart is provided for the determination of the ratio of hydraulic conductivity to specific yield in an aquifer for engineering applications.

\section{Governing Equation for Transient Groundwater Flows with a Moving Boundary}

Generally speaking, the governing equation for one-dimensional, lateral, unconfined groundwater flows in the Boussinesq equation (Parlange et al. 1984) is

$$
\frac{\partial h}{\partial t}=\frac{K}{S} \frac{\partial}{\partial x}\left(h \frac{\partial h}{\partial x}\right)
$$

in which $h=$ groundwater table height from the horizontal, impermeable aquifer base $[\mathrm{L}] ; K=$ hydraulic conductivity $\left[\mathrm{LT}^{-1}\right]$; $S=$ specific yield; $x=$ horizontal coordinate $[\mathrm{L}]$; and $t=$ time $[\mathrm{T}]$. Without loss of generality, the replenishment due to rainfall infiltration is not taken into account because our attention at present is mainly focused on the effects of reservoir drawdown. Moreover, the capillary effects on the groundwater table elevation are neglected as well. The modifications of this equation to incorporate vertical flow and capillary effects were previously made by Nielsen et al. (1997) and Parlange and Brutsaert (1987), respectively.

Meanwhile, the boundary and initial conditions for a semiinfinite aquifer in a slope look like

$$
\begin{gathered}
h[X(t), t]=H_{1}(t) \quad X(t)=H_{1}(t) \cot \beta, \quad t \geq 0 \\
h(\infty, t)=h_{i} \quad t \geq 0 \\
h(x, 0)=h_{i} \quad \infty>x \geq H_{1}(0) \cot \beta
\end{gathered}
$$

in which $X(t)=x$-coordinate of the moving boundary [L], with the origin of the $x$-coordinate located at the toe of the sloped interface; $\beta=$ slope angle; $h_{i}=$ initial height of the groundwater table across the aquifer [L]; and $H_{1}(t)=$ reservoir water level at the left boundary [L]. Eq. (2) actually implies that the seepage face is totally negligible. In reality, the groundwater flow in the adjacent aquifer may not instantly follow the variation of the water surface in the reservoir, thus resulting in the formation of a seepage face on the slope if the reservoir water level drops quickly enough or seepage flows move slowly enough. The intersection of the groundwater table and a slope surface is commonly termed the "exit point," and the region between the exit point and the reservoir water level is termed the "seepage face." In the present study, we assume that the exit point happens to be at the water surface of the reservoir, namely, the effect of the seepage face is neglected for the sake of convenient manipulation. Fig. 1 depicts an idealized cross section of the model under consideration.

Then, we prefer first to consider the drawdown condition at a constant speed; that is, the reservoir water level can be specified as

$$
H_{1}(t)=h_{i}-V t
$$

in which $V=$ drawdown speed of reservoir water level. When $V t$ is larger than $h_{i}$, the reservoir water level is below the data chosen for this problem and the moving boundary in Eq. (2) is no longer satisfied, which is out of the scope of the present paper. This means that in the present paper, $V t$ should always be no larger than $h_{i}$.

Similar to $\mathrm{Li}$ et al. (2000), we introduce a new variable $z=x-X(t)$, indicating the horizontal distance between a point in the aquifer and the intersection of reservoir water-surface level and sloped interface. Eqs. (1)-(4) can then be rewritten as

$$
\begin{gathered}
\frac{\partial h}{\partial t}=\frac{K}{S} \frac{\partial}{\partial z}\left(h \frac{\partial h}{\partial z}\right)-v(t) \frac{\partial h}{\partial z} \\
h(0, t)=h_{i}-V t \quad t \geq 0 \\
h(\infty, t)=h_{i} \quad t \geq 0 \\
h(z, 0)=h_{i} \quad \infty>z \geq 0
\end{gathered}
$$

in the new coordinate system, in which 


$$
v(t)=-\frac{d X(t)}{d t}=V \cot \beta
$$

denotes the horizontal speed of the intersection of the reservoir water level and the sloped interface during reservoir drawdown. In this way, we have reformulated a moving boundary problem of Eq. (1) into a fixed boundary problem. However, the governing equation now should include an additional advective term due to the effect of the sloped interface.

\section{Solution of the Linearized Boussinesq Equation}

If we assume that the variations of the groundwater table are small compared to a characteristic height of the groundwater table (van de Giesen et al. 1994; Serrano et al. 2007), the Boussinesq equation can be linearized and solved analytically. Eq. (6) is linearized by replacing the term $h$, associated with diffusive terms, with $h_{m}$, the average height of groundwater table, or $h_{i}$ in this study, and then can be written as

$$
\frac{\partial h}{\partial t}=\frac{K h_{i}}{S} \frac{\partial^{2} h}{\partial z^{2}}-V \cot \beta \frac{\partial h}{\partial z}
$$

We further assume that $u(z, t)=h_{i}-h(z, t)$ and Eqs. (7)-(11) can be transformed to

$$
\begin{gathered}
\frac{\partial u}{\partial t}=\frac{K h_{i}}{S} \frac{\partial^{2} u}{\partial z^{2}}-V \cot \beta \frac{\partial u}{\partial z} \\
u(0, t)=V t \quad t \geq 0 \\
u(\infty, t)=0 \quad t \geq 0 \\
u(z, 0)=0 \quad \infty>z \geq 0
\end{gathered}
$$

By using the Laplace transformation

$$
U(z, p)=\mathrm{L}[u(z, t)]=\int_{0}^{\infty} u(z, t) e^{-p t} d t
$$

Eq. (12) can be transformed into

$$
p U=\frac{K h_{i}}{S} \frac{d^{2} U}{d x^{2}}-V \cot \beta \frac{d U}{d x}
$$

The boundary conditions can be redefined in the Laplace domain as

$$
\left.U\right|_{x=0}=\left.\frac{V}{p^{2}} \quad U\right|_{x=\infty}=0
$$

The solution of Eq. (17) turns out to be

$$
U(z, p)=\frac{V}{p^{2}} \exp \left(z \frac{V \cot \beta-\sqrt{V^{2} \cot ^{2} \beta+4 \frac{K h_{i}}{S} p}}{2 \frac{K h_{i}}{S}}\right)
$$

By using the inverse Laplace transformation, $u(z, t)$ is now given by

$$
u(z, t)=V \int_{0}^{t} \frac{(-p+t) z \exp \left(\frac{-V^{2} p^{2} S \cot ^{2} \beta-S z^{2}+2 V p S z \cot \beta}{4 K h_{i} p}\right)}{2 \sqrt{\frac{\pi S p^{3}}{K h_{i}}}} d p
$$

and the height of groundwater table by

$$
h(z, t)=h_{i}-u(z, t)
$$

This analytic solution representing groundwater table variation in the adjacent aquifer during reservoir drawdown looks too complicated to be easily used for practical engineering. If we let $p$ in the first term of the exponential term $\left(-V^{2} p^{2} S \cot ^{2} \beta\right) / 4 K h_{i} p$ be 0 or $t$, two simpler approximate solutions can be derived. Because they are less and larger than the solution to Eq. (21), respectively, they represent the lower and upper bound solutions of the actual groundwater table height.

The lower solution now is

$$
\begin{aligned}
h_{l}(z, t)= & h_{i}-V t\left[\left(1+2 \lambda^{2}\right) \operatorname{erfc}(\lambda)-\frac{2}{\sqrt{\pi}} \lambda e^{-\lambda^{2}}\right] \\
& \times \exp \left(\frac{2 V t \cot \beta}{z} \lambda^{2}\right)
\end{aligned}
$$

And the upper solution is

$$
\begin{aligned}
h_{u}(z, t)= & h_{i}-V t\left[\left(1+2 \lambda^{2}\right) \operatorname{erfc}(\lambda)-\frac{2}{\sqrt{\pi}} \lambda e^{-\lambda^{2}}\right] \\
& \times \exp \left[\left(\frac{2 z-V t \cot \beta}{z^{2}} V t \cot \beta\right) \lambda^{2}\right]
\end{aligned}
$$

in which $\lambda=(z / 2) \sqrt{S /\left(K h_{i} t\right)} ; \operatorname{erfc}(\lambda)=(2 / \sqrt{\pi}) \int_{\lambda}^{\infty} e^{-x^{2}} d x$.

If we assume $M(\lambda)=\left(1+2 \lambda^{2}\right) \operatorname{erfc}(\lambda)-2 \lambda e^{-\lambda^{2}} / \sqrt{\pi}$, for engineering applications, a simpler polynomial can be used to fit $M(\lambda)$ (Zheng et al. 2005).

$$
M(\lambda)= \begin{cases}0.1091 \lambda^{4}-0.7501 \lambda^{3}+1.9283 \lambda^{2}-2.2319 \lambda+1 & 0 \leq \lambda<2 \\ 0 & \lambda \geq 2\end{cases}
$$

The lower and upper solutions of the height of the groundwater table are now given by

$$
\begin{aligned}
& h_{l}(z, t)=h_{i}-V t M(\lambda) \exp \left[\left(\frac{2 V t \cot \beta}{z}\right) \lambda^{2}\right] \\
& h_{u}(z, t)=h_{i}-V t M(\lambda) \exp \left[\left(\frac{2 z-V t \cot \beta}{z^{2}} V t \cot \beta\right) \lambda^{2}\right]
\end{aligned}
$$

If the upper and lower solutions are very close to each other, which will subsequently be proved in a few examples, both of them will be very close to the exact solution and can be regarded as accurate approximate solutions. Eqs. (24) and (25) indicate that the influential distance at a given time $t$ is $z=4 \sqrt{K h_{i} t / S}$. To rephrase the solution in the $x$-coordinate, it suffices only to substitute $z=x-X(t)$ into Eq. (25). 


\section{Numerical Verification and Discussion}

The Boussinesq equation describes nearly horizontal flows with the vertical motion and capillary effects of fluid neglected. The linearized Boussinesq equation further requires a constant transmissivity $T$, which can be written as

$$
T=K_{h} d
$$

for horizontal flows with a saturated aquifer thickness $d$ and horizontal hydraulic conductivity $K_{h}$. Hence, the transmissivity, which is directly proportional to the horizontal hydraulic conductivity and the saturated thickness of an aquifer, can be regarded as a measure of water seepage capacity horizontally. As $x$ increases, the hydraulic-gradient and groundwater-table variations drop and the transmissivity tends to a constant. As a result, the accuracy of the linearization for the Boussinesq equation is improved.

The effectiveness of the approximate solution is verified by comparing the upper solution with numerical results. The numerical calculation of the nonlinear Bousinesq equation is performed on the basis of a standard explicit finite difference scheme with high spacial resolution and small time step as follows:

$$
\begin{aligned}
\frac{h_{i+1, j}-h_{i, j}}{\Delta t}= & \frac{K}{S} h_{i, j} \frac{h_{i, j+1}-2 h_{i, j}+h_{i, j-1}}{\Delta z^{2}}+\frac{K}{S}\left(\frac{h_{i, j+1}-h_{i, j-1}}{2 \Delta z}\right)^{2} \\
& -v(t) \frac{h_{i, j+1}-h_{i, j-1}}{2 \Delta z}
\end{aligned}
$$

in which $\Delta t=$ time interval and $\Delta z=$ space increment. The subscripts $i$ and $j$ indicate time and space steps, respectively. The boundary conditions are Eqs. (7) and (8), and the initial condition is given by Eq. (9). Although such a numerical scheme is inefficient and restrictive in terms of the allowable time step due to stability consideration, it is still appropriate for our purpose. In the calculations of the solutions presented, $\Delta t$ is 0.0001 days and $\Delta z$ is $0.5 \mathrm{~m}$, and the total time $t$ is 5 days. Fig. 2 shows comparisons between the upper polynomial solutions and the numerical results for different drawdown speeds, hydraulic conductivities, specific yields, and slope angles, respectively. The seepage face is negligible in these simulations. The simulations show that the upper polynomial solution compares reasonably well with the numerical one. Fig. 2(a) illustrates that the elevation of the groundwater table is lower as the drawdown speed increases. Fig. 2(b) shows the
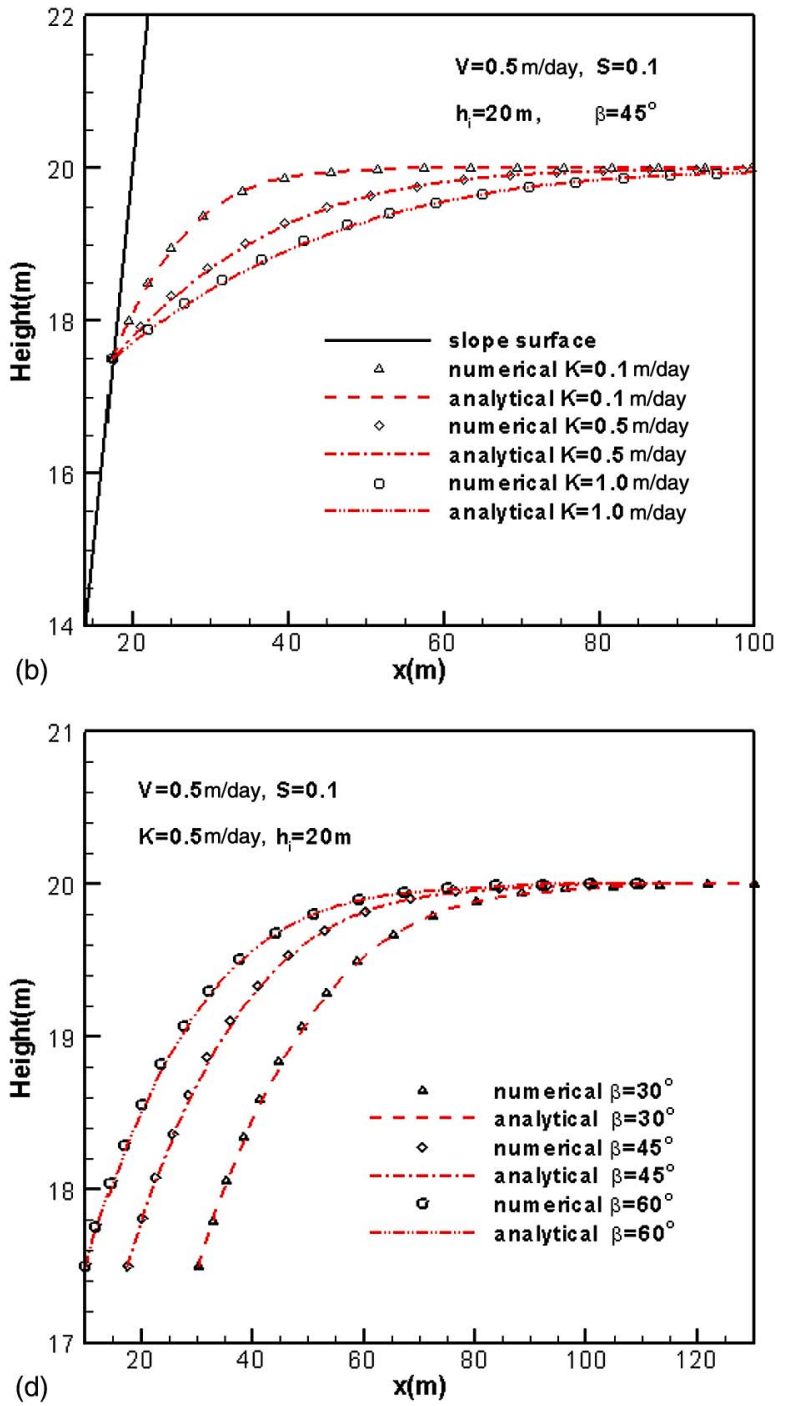

Fig. 2. Comparison between upper polynomial solutions and explicit finite-difference solutions for (a) different drawdown speeds: $0.1 \mathrm{~m} /$ day, $0.5 \mathrm{~m} /$ day, and $1.0 \mathrm{~m} /$ day; (b) different hydraulic conductivities: $0.1 \mathrm{~m} /$ day, $0.5 \mathrm{~m} /$ day, and $1 \mathrm{~m} /$ day; (c) different specific yields: 0.05 , 0.1 , and 0.5 ; (d) different slope angles: $30^{\circ}, 45^{\circ}$, and $60^{\circ}$ 
groundwater table heights for different hydraulic conductivities, demonstrating that as the hydraulic conductivity increases, the influential region of reservoir drawdown becomes larger, and hence the groundwater table is lower. In contrast, Fig. 2(c) indicates that the aquifer has a higher groundwater level for smaller specific yield. Fig. 2(d) displays the groundwater table variation for different slope angles. Obviously, the groundwater table for a mild slope is lower than that for a steeper one.

To quantitatively estimate the accuracy of the upper polynomial solution, we define

$$
\text { accuracy ratio in percent }=\frac{\left|h_{u}-h_{n}\right|}{h_{i}} \times 100
$$

in which $h_{u}$ and $h_{n}$ are the groundwater table heights for upper polynomial and numerical solutions, respectively, and $h_{i}$ is the initial groundwater table height. We have calculated the accuracy ratios for all previously mentioned simulations, as shown in Fig. 3. The results prove that the accuracy ratios are less than $0.8 \%$, which is precise enough for engineering applications. As $x$ increases, the differences of all the simulations exhibit similar variation patterns.
The reason is that the difference between fitted polynomial and actual function of $M(\lambda)$ has the similar variation pattern. Fig. 3(a) presents the accuracy change with the drawdown speed, showing that the maximal error increases with the drawdown speed. For instance, the maximal error is only $0.03 \%$ for a drawdown speed of $0.1 \mathrm{~m} /$ day. In contrast, the maximal error rises up to $0.79 \%$ for a drawdown speed of $1 \mathrm{~m} /$ day. Fig. 3(b) demonstrates that the maximal error grows from $0.13 \%$ to $0.24 \%$ as the hydraulic conductivity increases from $0.1 \mathrm{~m} /$ day to $1.0 \mathrm{~m} /$ day. Fig. 3(c) illustrates that the maximal error decreases from $0.24 \%$ to $0.13 \%$ as the specific yield varies from 0.05 to 0.5 . Fig. 3 (d) shows that the maximal error increases as the reservoir-aquifer interface angle becomes larger. As the slope angle expands from $30^{\circ}$ to $60^{\circ}$, the maximal error increases from $0.18 \%$ to $0.24 \%$. In addition, we also have calculated the differences between the upper and lower polynomial solutions for all the previously mentioned simulations to validate our approximate solutions. The results show that the maximal difference between two polynomial solutions is minor, approximately of the order of $0.3 \%$ of the initial groundwater table height, implying that both upper and lower solutions can be regarded as accurate approximate solutions.
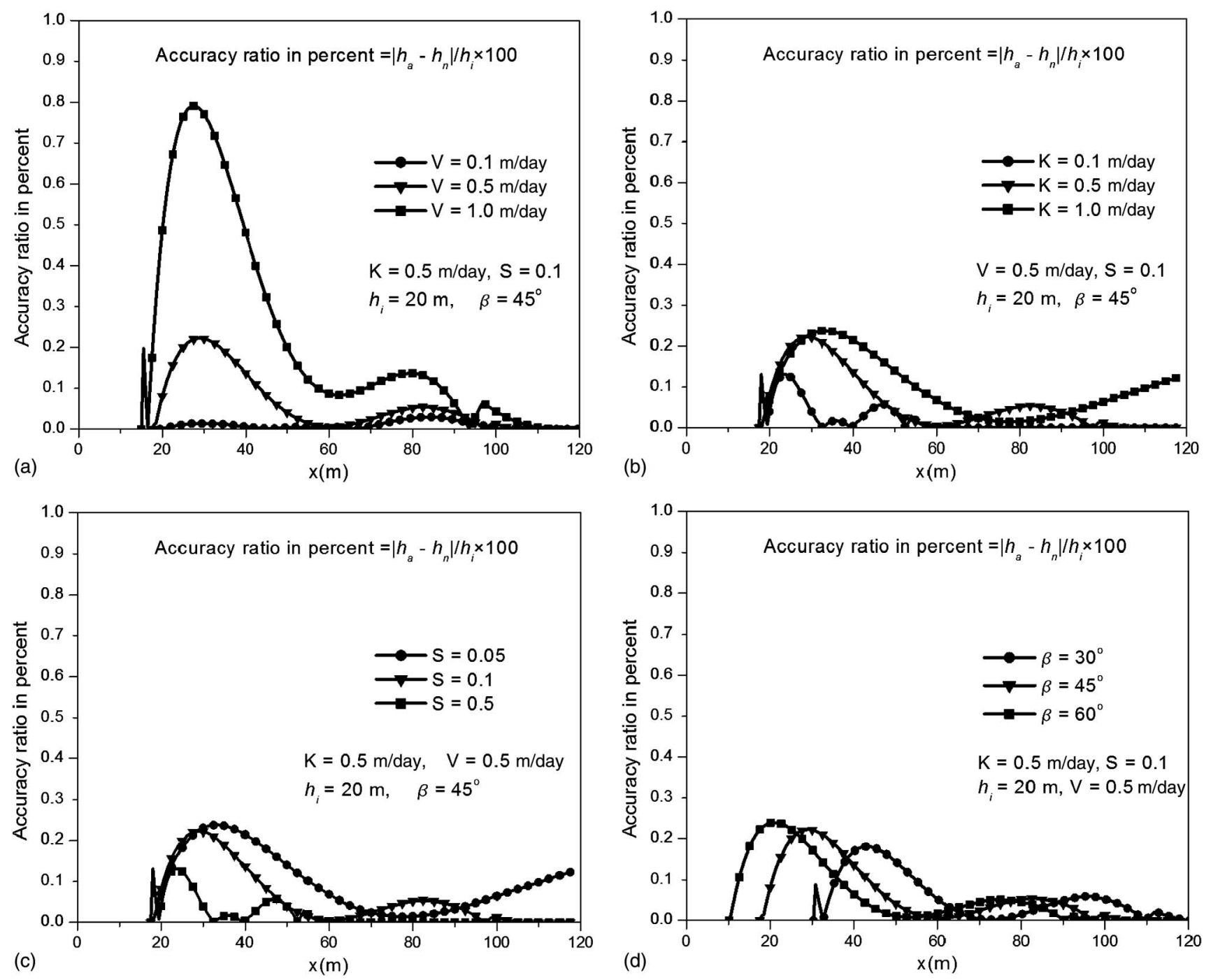

Fig. 3. Quantitative differences of upper polynomial solutions and explicit finite-difference solutions for (a) different drawdown speeds: $0.1 \mathrm{~m} /$ day, $0.5 \mathrm{~m} /$ day, and $1 \mathrm{~m} /$ day; (b) different hydraulic conductivities: $0.1 \mathrm{~m} /$ day, $0.5 \mathrm{~m} /$ day, and $1 \mathrm{~m} /$ day; (c) different specific yields: 0.05 , 0.1, and 0.5; (d) different slope angles: $30^{\circ}, 45^{\circ}$, and $60^{\circ}$ 


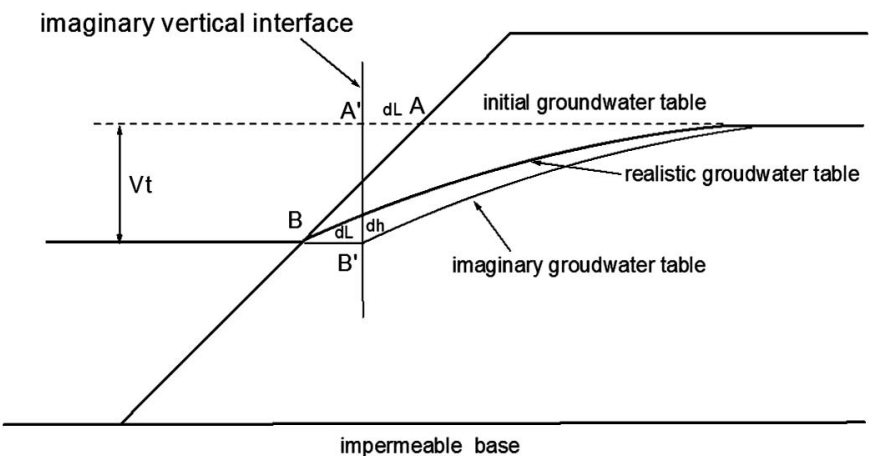

impermeable base

Fig. 4. Schematic diagram of the difference between the "vertical interface solution" and the realistic upper polynomial solution

Most existing solutions (e.g., Zheng et al. 2005) were derived on the basis of the assumption of a vertical reservoir-aquifer interface. Simplifying the sloped interface by a vertical boundary may cause some errors. The difference between the "vertical interface solution" and the more realistic upper polynomial solution is exhibited in Fig. 4. The "vertical interface solution" matches the reservoir water level along the imaginary vertical interface, and thus coincides with the initial reservoir water level at point $A^{\prime}$ and the current reservoir water level at point $B^{\prime}$. The upper polynomial solution matches the reservoir water level along the sloped interface, and hence coincides with the initial reservoir water level at point $A$ and the current reservoir water level at point $B$. The imaginary vertical interface is assumed to pass through the midpoint of the line $A B$ (Nielsen 1990), indicating that this imaginary interface is moving leftward during the process of reservoir drawdown. The maximal difference $d h$ between the "vertical interface solution" and the more realistic upper polynomial solution occurs along the imaginary vertical interface, as shown in Fig. 4. Furthermore, Fig. 5 illustrates that the errors caused by vertical-interface assumption increase with the reservoir drawdown height and decrease with the slope angle. It should be noted that simplifying the sloped reservoir-aquifer interface by a vertical boundary may

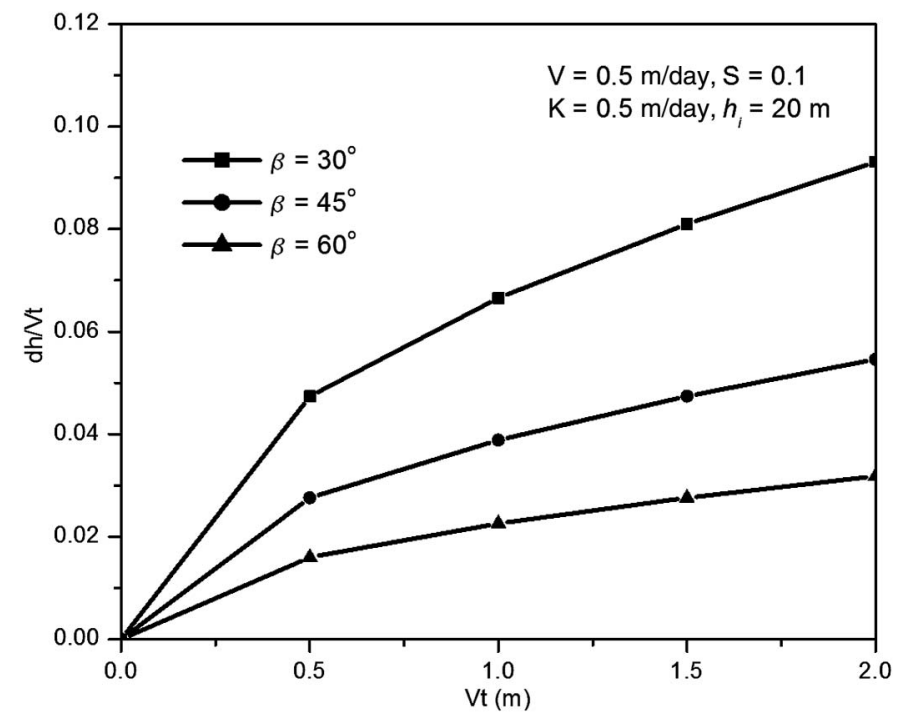

Fig. 5. Difference between the "vertical interface solution" and the realistic upper polynomial solution for different slope angles: $30^{\circ}$, $45^{\circ}$, and $60^{\circ}$ lead to an error of up to $10 \%$ of the height of the reservoir drawdown (Fig. 5).

One problem in using the Boussinesq equation is that no criterion is given for the estimation of seepage-face development. In the present paper, the seepage face is assumed to be entirely negligible. Turner (1993) proposed a model for the movement of the exit point entirely on the basis of the dynamics of an isolated water particle on the seepage face. On the basis of the concept from Turner, the following criterion can be derived to predict situations in which the assumption of a negligible seepage face is reasonable:

$$
\frac{K \sin ^{2} \beta}{V S} \geq 1
$$

This expression implies that the approximate polynomial solutions are only suitable for relatively slow drawdown speed, small specific yield, high hydraulic conductivity, or a steep reservoiraquifer interface. The previously mentioned criterion limits the application of the approximate polynomial solutions. If the seepage face occurs, the approximate polynomial solutions are probably impractical and may underestimate the height of the groundwater table; however, the approximate polynomial solutions are both simple and very useful if the seepage face can be negligible.

Eqs. (24) and (25) indicate that the influential distance at any time $t$ can be measured by $z=4 \sqrt{K h_{i} t / S}$. Then, this relationship can be used to determine the aquifer parameters. Assume that the initial groundwater table in the aquifer is horizontal and equals the initial reservoir water level. A monitoring well is drilled deep into the aquifer. Evidently, we can obtain the distance $L$ between the monitoring well and the intersection of the reservoir water level and the sloped interface at the time $t$ when the groundwater table in the monitoring well begins to drop (Fig. 6). The ratio of hydraulic conductivity $K$ to specific yield $S$ can then be determined by

$$
\frac{K}{S}=\frac{\mathrm{L}^{2}}{16 t h_{i}}
$$

Eq. (30) for any sloped reservoir-aquifer interface and a chart as presented in Fig. 6 are both suitable for engineering applications. Although the chart is valid only for conditions as shown in Fig. 6, it could be useful for a preliminary estimate of the ratio of hydraulic conductivity to specific yield in an aquifer.

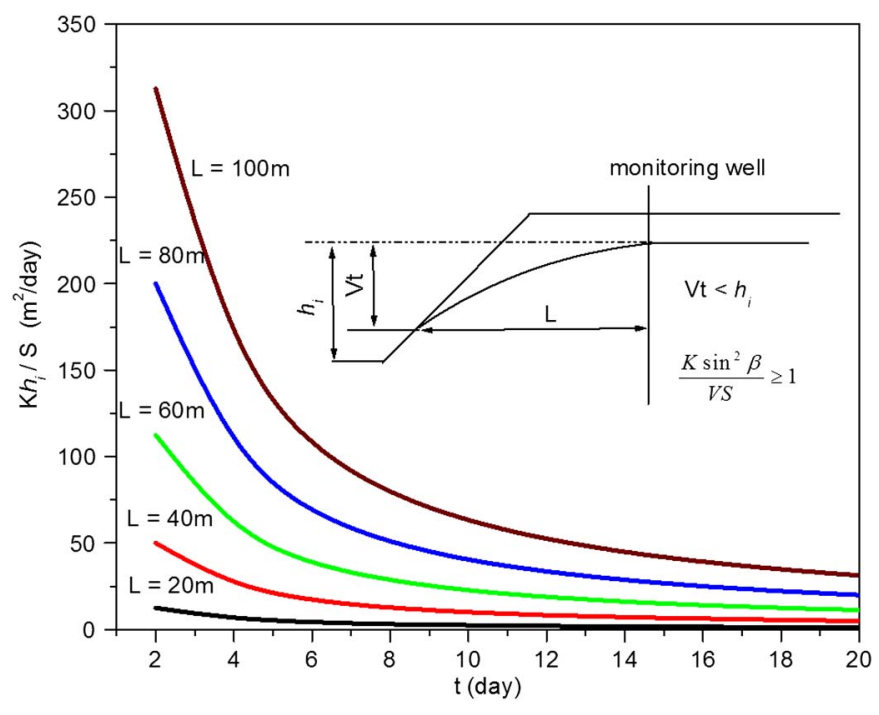

Fig. 6. Chart for the determination of aquifer hydraulic parameters 


\section{Conclusions}

An approximate analytical solution is proposed by solving the linearized Boussinesq equation for one-dimensional groundwater flows in a slant slope adjacent to a reservoir during water drawdown. A constant drawdown speed, a negligible seepage face, and a moving boundary condition are assumed. Since the analytical solution we have yielded is difficult to use for practical engineering, it is simplified to upper- and lower-bound solutions and further fitted in the form of an approximate polynomial formula. The upper polynomial solution is satisfactorily verified by comparison with a number of numerical simulations. The results indicate that the effect of slope angle is significant and the replacement of slant slope by a vertical interface may cause errors of up to $10 \%$ in the prediction of groundwater elevation. On the basis of the polynomial equations, a methodology along with a useful chart is provided for the determination of the ratio of hydraulic conductivity to specific yield. We should emphasize at this moment that the upper and lower polynomial solutions with seepage face entirely neglected are only applicable to the cases for relatively slow drawdown speed, small specific yield, high hydraulic conductivity, or a steep reservoir-aquifer interface. On the other hand, it appears to be very helpful for a preliminary estimate of the ratio of hydraulic conductivity to specific yield. Even for more complicated scenarios when we have to resort to more general numerical approaches, the theoretical expressions derived in this paper seem to be useful in the validation and verification of numerical models and schemes.

\section{Acknowledgments}

This research was supported by the National Natural Science Foundation of China (Grant Nos. 10825211, 10932012) and the Knowledge Innovation Project of the Chinese Academy of Sciences of China (Grant No. KJCX2-SW-L1-4). The writers are very thankful to the referees for their valuable comments and suggestions to improve the quality of this paper.

\section{References}

Bansal, R. K., and Das, S. K. (2010). "Analytical study of water table fluctuation in unconfined aquifer due to varying bed slopes and spatial location of the recharge basin." J. Hydrol. Eng., 15(11), 909-917.

Heaslet, M. A., and Alksne, A. (1961). "Diffusion from a fixed surface with a concentration-dependent coefficient." J. Soc. Ind. Appl. Math., 9(4), 584-596.

Hogarth, W. L., Parlange, J.-Y., Parlange, M. B., and Lockington, D. (1999). "Approximate analytical solution of the Boussinesq equation with numerical validation." Water Resour. Res., 35(10), 3193-3197.

Li, L., Barry, D. A., Stagnitti, F., Parlange, J.-Y. , and Jeng, D.-S. (2000). "Beach water table fluctuations due to spring-neap tides: Moving boundary effects." Adv. Water Resour., 23(8), 817-824.

Lockington, D. A., Parlange, J.-Y., Parlange, M. B., and Selker, J. (2000). "Similarity solution of the Boussinesq equation." Adv. Water Resour., 23(7), 725-729.

Mizumura, K. (2009). "Approximate solution of nonlinear Boussinesq equation.” J. Hydrol. Eng., 14(10), 1156-1164.

Nielsen, P. (1990). "Tidal dynamics of the water table in beaches." Water Resour. Res., 26(9), 2127-2134.

Nielsen, P., Fenton, J. D., Aseervathan, R. A., and Perrochet, P. (1997). "Watertable waves in aquifers of intermediate depths." Adv. Water Resour., 20(1), 37-43.

Parlange, J.-Y, and Brutsaert, W. (1987). "A capillary correction for free surface flow of groundwater." Water Resour. Res., 23(5), $805-808$

Parlange, J.-Y, Hogarth, W. L., Govindaraju, R. S., Parlange, M. B., and Lockington, D. A. (2000). "On an exact analytical solution of the Boussinesq equation." Transp. Porous Media, 39(3), 339-345.

Parlange, J.-Y, Stagnitti, F., Starr, J. L., and Braddock, R. D. (1984). "Freesurface flow in porous media and periodic solution of the shallow-flow approximation." J. Hydrol. (Amsterdam), 70(1-4), 251-263.

Serrano, S. E., Workman, S. R., Srivastava, K., and Cleave, B. M. V. (2007). "Models of nonlinear stream aquifer transients." J. Hydrol. (Amsterdam), 336(1-2), 199-205.

Song, Z. Y., Li, L., Kong, J., and Zhang, H. G. (2007). "A new analytical solution of tidal water table fluctuations in a coastal unconfined aquifer." J. Hydrol. (Amsterdam), 340(3-4), 256-260.

Telyakovskiy, A. S., and Allen, M. B. (2006). "Polynomial approximate solutions to the Boussinesq equation." Adv. Water Resour., 29(12), 1767-1779.

Telyakovskiy, A. S., Braga, G. A., Kurita, S., and Mortensen, J. (2010). "On a power series solution to the Boussinesq equation." Adv. Water Resour., 33(9), 1128-1129.

Turner, I. (1993). "Water table outcropping on macro-tidal beaches: A simulation model." Mar. Geol., 115(3-4), 227-238.

van de Giesen, N. C., Parlange, J. Y., and Steenhuis, T. S. (1994). "Transient flow to open drains: comparison of linearized solutions with and without the Dupuit assumption." Water Resour. Res., 30(11), 3033-3039.

Zheng, Y. R., Shi, W. M., Kong, W. X., and Lei, W. J. (2005). "Determination of the phreatic-line under reservoir drawdown condition." Proc., Sessions of the Geo-Frontiers 2005 Congress, M. A. Gabr, J. J. Bowders, D. Elton and J. G. Zornberg, eds., ASCE, Reston, VA. 